\title{
Quantum correlations in two-fermion systems
}

\author{
John Schliemann, ${ }^{1}$ J. Ignacio Cirac, ${ }^{2}$ Marek Kuś, ${ }^{3}$ Maciej Lewenstein, ${ }^{4}$ and Daniel Loss ${ }^{5}$ \\ ${ }^{1}$ Department of Physics, The University of Texas, Austin, Texas 78712 \\ ${ }^{2}$ Institut für Theoretische Physik, Universität Innsbruck, A-6020 Innsbruck, Austria \\ ${ }^{3}$ Centre for Theoretical Physics, Polish Academy of Sciences, 02668 Warsaw, Poland \\ ${ }^{4}$ Institut für Theoretische Physik, Universität Hannover, 30167 Hannover, Germany \\ ${ }^{5}$ Department of Physics and Astronomy, University of Basel, CH-4056 Basel, Switzerland
}

(Received 18 December 2000; published 3 July 2001)

\begin{abstract}
We characterize and classify quantum correlations in two-fermion systems having $2 K$ single-particle states. For pure states we introduce the Slater decomposition and rank (in analogy to Schmidt decomposition and rank); i.e., we decompose the state into a combination of elementary Slater determinants formed by pairs of mutually orthogonal single-particle states. Mixed states can be characterized by their Slater number which is the minimal Slater rank required to generate them. For $K=2$ we give a necessary and sufficient condition for a state to have a Slater number 1 . We introduce a correlation measure for mixed states which can be evaluated analytically for $K=2$. For higher $K$, we provide a method of constructing and optimizing Slater number witnesses, i.e., operators that detect Slater numbers for some states.
\end{abstract}

DOI: 10.1103/PhysRevA.64.022303

PACS number(s): 03.65.Ta, 89.70.+c

\section{INTRODUCTION}

In recent years a lot of effort [1,2] in quantum information theory (QIT) has been devoted to the characterization of entanglement, which is one of the key features of quantum mechanics [3]. The resources needed to implement a particular protocol of quantum information processing (see, e.g., [4]) are closely linked to the entanglement properties of the states used in the protocol. In particular, entanglement lies at the heart of quantum computing [3]. The most fundamental question with regard to entanglement is, given a state of a multiparty system, is it entangled or not (i.e., is it separable [5])? If the answer is yes, then the next question is how strong the entanglement is. For pure states in bipartite systems the latter question can be answered by looking at the Schmidt decomposition [6], i.e., the decomposition of the vector in a product basis of the Hilbert space with a minimal number of terms. For mixed states already the first question is notoriously hard to answer. There exist, however, many separability criteria, such as the Peres-Horodecki criterion $[7,8]$ and more recent concepts such as entanglement witnesses and the corresponding "entanglement revealing" positive maps $[9,10]$.

While entanglement plays an essential role in quantum communication between parties separated by macroscopic distances, the characterization of quantum correlations at short distances is also an open problem, which has received much less attention so far. In this case the indistinguishable character of the particles involved (electrons, photons, etc.) has to be taken into account. In his classic book, Peres [6] discussed the entanglement in elementary states of indistinguishable particles. These are symmetrized or antisymmetrized product states for bosons and fermions, respectively. It is easy to see that all such states of two-fermion systems, and as well as such states formed by two noncollinear singleparticle states in two-boson systems, are necessarily entangled in the usual sense. However, in the case of particles far apart from each other, this type of entanglement is not of physical relevance: "No quantum prediction, referring to an atom located in our laboratory, is affected by the mere presence of similar atoms in remote parts of the universe" [6]. This kind of entanglement between indistinguishable particles being far apart from each other is not the subject of this paper. Our aim here is rather to classify and characterize the quantum correlations between indistinguishable particles (in our case fermions) at short distances. We discuss below why this problem is relevant for quantum information processing in various physical systems.

For indistinguishable particles a pure quantum state must be formulated in terms of Slater determinants or Slater permanents for fermions and bosons, respectively. Generically, a Slater determinant contains correlations due to the exchange statistics of the indistinguishable fermions. As the simplest possible example, consider a wave function of two (spinless) fermions,

$$
\Psi\left(\vec{r}_{1}, \vec{r}_{2}\right)=\frac{1}{\sqrt{2}}\left[\phi\left(\vec{r}_{1}\right) \chi\left(\vec{r}_{2}\right)-\phi\left(\vec{r}_{2}\right) \chi\left(\vec{r}_{1}\right)\right],
$$

with two orthonormalized single-particle wave functions $\phi(\vec{r})$ and $\chi(\vec{r})$. Operator matrix elements between such single Slater determinants contain terms due to the antisymmetrization of coordinates ("exchange contributions" in the language of Hartree-Fock theory). However, if the moduli of $\phi(\vec{r})$ and $\chi(\vec{r})$ have only vanishingly small overlap, these exchange correlations will also tend to zero for any physically meaningful operator. This situation is generically realized if the supports of the single-particle wave functions are essentially centered around locations being sufficiently apart from each other or the particles are separated by a sufficiently large energy barrier. In this case the antisymmetrization present in Eq. (1) has no physical effect.

Such observations clearly justify the treatment of indistinguishable particles separated by macroscopic distances as effectively distinguishable objects. So far, research in quantum information theory has concentrated on this case, where the 
exchange statistics of particles forming quantum registers could be neglected or was not specified at all.

The situation is different if the particles constituting, say, qubits are close together and possibly coupled in some computational process. This the case for all proposals of quantum information processing based on quantum dot technology [11-13]. Here qubits are realized by the spins of electrons residing in a system of quantum dots. The electrons have the possibility of tunneling eventually from one dot to the other with a probability which can be modified by varying external parameters such as gate voltages and magnetic field. In such a situation the fermionic statistics of electrons is clearly essential.

Additional correlations in many-fermion systems arise if more than one Slater determinant is involved, i.e., if there is no single-particle basis such that a given state of $N$ indistinguishable fermions can be represented as an elementary Slater determinant (i.e., fully antisymmetric combination of $N$ orthogonal single-particle states). These correlations are the analog of quantum entanglement in separated systems and are essential for quantum information processing in nonseparated systems.

As an example consider a "swap" process exchanging the spin states of electrons on coupled quantum dots by gating the tunneling amplitude between them $[12,13]$. Before the gate is turned on, the two electrons in the neighboring quantum dots are in a state represented by a simple Slater determinant and can be regarded as distinguishable since they are separated by a large energy barrier. When the barrier is lowered, more complex correlations between the electrons due to the dynamics arise. Interestingly, as shown in Refs. [12], [13], during such a process the system must necessarily enter a highly correlated state that cannot be represented by a single Slater determinant. The final state of the gate operation, however, is, similarly as the initial one, essentially given by a single Slater determinant. Moreover, by adjusting the gating time appropriately one can also perform a "square root of a swap" which turns a single Slater determinant into a "maximally" correlated state in much the same way [13]. At the end of such a process the electrons can again be viewed as effectively distinguishable, but are in a maximally entangled state in the usual sense of distinguishable separated particles. In this sense the highly correlated intermediate state can be viewed as a resource for the production of entangled states.

We expect that similar scenarios apply to other schemes of quantum information processing that involve cold particles (bosons or fermions) interacting at microscopic distances at which the quantum statistics becomes essential. For instance, it should be of relevance for quantum computing models employing ultracold atoms in optical lattices [14] or ultracold atoms in arrays of optical microtraps [15].

It is the purpose of the present paper to analyze the above type of quantum correlations between indistinguishable fermions in more detail. However, to avoid confusion with the existing literature we shall reserve in the following the term "entanglement" for separated systems and characterize the analogous quantum correlation phenomenon in nonseparated fermionic systems by the notions of Slater rank and Slater number to be defined below.

We are going to formulate analogies with the theory of entanglement and translate several very recent results $[10,16,17]$ concerning standard systems of distinguishable parties (Alice $\neq \mathrm{Bob}$ ) to the case of indistinguishable fermions. In general, we will deal with a system of two fermions each of which live in a $2 K$-dimensional single-particle space.

The plan of the paper is as follows: In Sec. II we discuss pure states and formulate the analog of Schmidt decomposition and rank-Slater decomposition and rank. We then discuss a simple operational criterion for the case of two electrons in two neighboring quantum dots $(K=2)$ to determine whether a given state is of Slater rank 1 . This criterion was first derived in Ref. [13]. In Sec. III we define the concept of a Slater number for mixed states. For $K=2$ we present the necessary and suficient condition for a mixed state to have the Slater number 1. This is an analog of the PeresHorodecki criterion $[7,8]$ in the Wootters formulation [18]. In Sec. IV we extend the results of Sec. III and define a Slater correlation measure which is the analog of the entanglement formation measure [19]. This quantity can be calculated analytically for the case $K=2$, in analogy to the Wootters result [18]. In Sec. V we turn to the case $K>2$ and introduce Slater number witnesses of canonical form (defined in analogy to entanglement $[9,16]$ and Schmidt number $[20,17]$ witnesses). We construct examples of such $k$-Slater witnesses, which provide the necessary conditions for a given state to have a Slater number smaller than $k$; we also discuss optimization of Slater witnesses. Finally, we analyze the associated [21] positive maps. We close by discussing further analogies, but also differences, between entanglement in separated systems of distinguishable particles as opposed to quantum correlations in nonseparated systems of indistinguishable particles.

\section{SLATER RANK OF PURE STATES}

We consider two indistinguishable fermions each of which resides in the single-particle Hilbert space $\mathcal{C}^{2 K}$. This situation is given, e.g., in a system of two electrons in $K$ neighboring quantum dots where only the orbital ground state of each dot is taken into account. Alternatively, one may think of, say, two quantum dots with an appropriate number of orbital states available for the two fermions.

The states (density matrices) in such a system are positive self-adjoint operators acting on the antisymmetric space $\mathcal{A}\left(\mathcal{C}^{2 K} \otimes \mathcal{C}^{2 K}\right)$. Let us first consider pure states, i.e., projectors on a vector $|\Psi\rangle \in \mathcal{A}\left(\mathcal{C}^{2 K} \otimes \mathcal{C}^{2 K}\right)$. Let $f_{a}, f_{a}^{\dagger}, a$ $=1, \ldots, 2 \mathrm{~K}$, denote the fermionic annihilation and creation operators of single-particle states forming an orthonormal basis in $\mathcal{C}^{2 K}$, and $|\Omega\rangle$ denotes the vacuum state. Each vector in the two-electron space can be represented as $|\Psi\rangle$ $=\Sigma_{a, b} w_{a b} f_{a}^{\dagger} f_{b}^{\dagger}|\Omega\rangle$, where $w_{a b}=-w_{b a}$ is an antisymmetric matrix. We have the following generalization of theorem 4.3.15 from Ref. [22], which will allow us to define the fermionic analog of the Schmidt decomposition:

Lemma 1. For any antisymmetric $N \times N$ matrix $A \neq 0$ there exists a unitary transformation $U^{\prime}$ such that $A$ 
$=U^{\prime} Z U^{\prime T}$, where the matrix $Z$ has blocks on the diagonal,

$$
Z=\operatorname{diag}\left[Z_{0}, Z_{1}, \ldots, Z_{M}\right], \quad Z_{0}=0, \quad Z_{i}=\left[\begin{array}{cc}
0 & z_{i} \\
-z_{i} & 0
\end{array}\right],
$$

and $Z_{0}$ is a $(N-2 M) \times(N-2 M)$ null matrix.

Proof. Let $A$ be an $N \times N$, complex, antisymmetric matrix acting on $\mathcal{C}^{N}, A=-A^{T}$; hence, $A^{\dagger}=-A^{*}$. Let us define $B$ $:=A A^{*}=-A A^{\dagger}$. Here $B$ is Hermitian, $B=B^{\dagger}$, and hence diagonalizable by a unitary transformation: $B=U D U^{\dagger}$, $U U^{\dagger}=1, D$-diagonal. Now consider $C:=U^{\dagger} A U^{*}$. It is easy to check that $C$ is antisymmetric, $C^{T}=-C$, and normal, $C C^{\dagger}=C^{\dagger} C$. Let us decompose $C$ into its real and imaginary parts: $C=F+i G ; F, G$ are real $N \times N$ matrices. Since $C$ is antisymmetric, so are $F$ and $G$. Since $C$ is normal, $F$ and $G$ commute. Thus $F$ and $G$ are real, antisymmetric, commuting matrices. Hence they can be simultaneously brought to block-diagonal forms by a real orthogonal transformation [22], $F=O F_{b d} O^{T}, G=O G_{b d} O^{T}$, where $O$ is an $N \times N$ matrix, $O O^{T}=I$, where

$$
\begin{aligned}
& F_{b d}=\operatorname{diag}\left[X_{0}, X_{1}, \ldots, X_{K}\right], \\
& G_{b d}=\operatorname{diag}\left[Y_{0}, Y_{1}, \ldots, Y_{L}\right],
\end{aligned}
$$

and $X_{0}, Y_{0}$ are null matrices of some dimensions, $X_{0}=0$, $Y_{0}=0$, whereas $X_{i}, Y_{i}$ are standard antisymmetric $2 \times 2$ blocks:

$$
X_{i}=\left[\begin{array}{cc}
0 & x_{i} \\
-x_{i} & 0
\end{array}\right], \quad Y_{i}=\left[\begin{array}{cc}
0 & y_{i} \\
-y_{i} & 0
\end{array}\right] .
$$

Thus $C=O Z O^{T}$ where $Z$ has the form (2) and, finally $A$ $=U C U^{T}=U O Z O^{T} U^{T}=(U O) Z(U O)^{T}=U^{\prime} Z U^{\prime T}$ with $U^{\prime}$ unitary.

Lemma 2. Every vector in antisymmetric space $\mathcal{A}\left(\mathcal{C}^{2 K} \otimes \mathcal{C}^{2 K}\right)$ can be represented in an appropriately chosen basis in $\mathcal{C}^{2 K}$ in a form of the Slater decomposition

$$
|\Psi\rangle=\frac{1}{\sqrt{\sum_{i=1}^{K}\left|z_{i}\right|^{2}}} \sum_{i=1}^{K} z_{i} f_{a_{1}(i)}^{\dagger} f_{a_{2}(i)}^{\dagger}|\Omega\rangle,
$$

where the states $f_{a_{1}(i)}^{\dagger}|\Omega\rangle, f_{a_{2}(i)}^{\dagger}|\Omega\rangle, i=1, \ldots, K$, form an orthonormal basis in $\mathcal{C}^{2 K}$; i.e., each of these single-particle states occurs only in one term in the summation (5). The number of nonvanishing coefficients $z_{i}$ (i.e., the number of elementary Slater determinants required to construct $|\Psi\rangle$ ) is called the Slater rank.

Proof. Let $|\Psi\rangle=\Sigma_{a, b} w_{a b} f_{a}^{\dagger} f_{b}^{\dagger}|\Omega\rangle$. Note that the change of basis in $\mathcal{C}^{2 K}$ corresponds to a unitary transformation of fermionic operators, $f_{a}^{\dagger}=\Sigma_{b} U_{b a}\left(f_{b}^{\prime}\right)^{\dagger}$, which implies that in the new basis $w^{\prime}=U w U^{T}$. From lemma 1 we may choose $U$ such that $w^{\prime}$ will have the form (2), which provides the Slater decomposition.

From the point of view of applications in quantum dot computers, it is important to be able to distinguish states with Slater rank 1 (which can be easily prepared and detected) from those that involve more than one elementary Slater de- terminant. In general, given $|\Psi\rangle$ in some basis, in order to check the Slater rank, one has to perform the Slater decomposition. As we know from Ref. [13], the situation is simpler for the case $K=2$, where we have the following.

Lemma 3 (Ref. [13]). A vector $|\Psi\rangle=\sum_{a, b=1}^{4} w_{a b} f_{a}^{\dagger} f_{b}^{\dagger}|\Omega\rangle$ in $\mathcal{A}\left(\mathcal{C}^{4} \otimes \mathcal{C}^{4}\right)$ has Slater rank 1 iff

$$
\eta(|\Psi\rangle)=\left|\sum_{a, b, c, d} \epsilon^{a b c d} w_{a b} w_{c d}\right|=0,
$$

where $\epsilon^{a b c d}$ denotes the totally antisymmetric tensor in $\mathcal{C}^{4} \otimes \mathcal{C}^{4} \otimes \mathcal{C}^{4} \otimes \mathcal{C}^{4}$.

Remark. The quantity $\eta(|\Psi\rangle)$ can be constructed from the dual state

$$
|\widetilde{\Psi}\rangle=\sum_{a, b} \widetilde{w}_{a b} f_{a}^{\dagger} f_{b}^{\dagger}|\Omega\rangle
$$

defined by the dual matrix

$$
\widetilde{w}_{a b}=\frac{1}{2} \sum_{c, d} \epsilon^{a b c d} w_{c d}^{*} .
$$

With these definitions we have

$$
\eta(|\Omega\rangle)=|\langle\widetilde{\Psi} \mid \Psi\rangle| .
$$

The proof of this lemma was presented first in Ref. [13]. An alternative proof can be given using lemma 1 and observing that

$$
\operatorname{det} w=\left(\frac{1}{8}\langle\widetilde{\Psi} \mid \Psi\rangle\right)^{2}
$$

where $w$ is the antisymmetric $4 \times 4$ matrix defining $|\Psi\rangle$.

In the Appendix we list some further useful properties of $\eta(|\Psi\rangle)$ and the relation of the dualization operation to an antiunitary implementation of particle-hole transformation. An interesting further question is possible generalizations of the above result to the case of $K$ fermions having a singleparticle space $\mathcal{C}^{2 K}$.

\section{SLATER NUMBER OF MIXED STATES}

Let us now generalize the concepts introduced above to the case of mixed states. To this end, we define the Slater number of a mixed state, in analogy to the Schmidt number for the case of distinguishable parties [20,17]

Definition 1. Consider a density matrix $\rho$ of a two-fermion system and all its possible convex decompositions in terms of pure states, i.e., $\rho=\Sigma_{i} p_{i}\left|\psi_{i}^{r_{i}}\right\rangle\left\langle\psi_{i}^{r_{i}}\right|$, where $r_{i}$ denotes the Slater rank of $\left|\psi_{i}^{r_{i}}\right\rangle$; the Slater number of $\rho, k$, is defined as $k=\min \left\{r_{\max }\right\}$, where $r_{\max }$ is the maximum Slater rank within a decomposition, and the minimum is taken over all decompositions.

In other words, $k$ is the minimal Slater rank of the pure states that are needed in order to construct $\rho$, and there is a construction of $\rho$ that uses pure states with Slater rank not exceeding $k$. 
Many of the results concerning Schmidt numbers can be transferred directly to the Slater number. For instance, let us denote the whole space of density matrices in $\mathcal{A}\left(\mathcal{C}^{2 K} \otimes \mathcal{C}^{2 K}\right)$ by $\mathrm{Sl}_{K}$ and the set of density matrices that have Slater number $k$ or less by $\mathrm{Sl}_{k}$. Here $\mathrm{Sl}_{k}$ is a convex compact subset of $\mathrm{Sl}_{K}$; a state from $\mathrm{Sl}_{k}$ will be called a state of (Slater) class $k$. Sets of increasing Slater number are embedded into each other, i.e., $\mathrm{Sl}_{1} \subset \mathrm{Sl}_{2} \subset \cdots \mathrm{Sl}_{k} \cdots \subset \mathrm{Sl}_{K}$. In particular, $\mathrm{Sl}_{1}$ is the set of states that can be written as a convex combination of elementary Slater determinants; $\mathrm{Sl}_{2}$ is the set of states of Slater number 2, i.e., those that require at least one pure state of Slater rank 2 for their formation, etc.

The determination of the Slater number of a given state is in general a very difficult task. Similarly, however, as in the case of separability of mixed states of two qubits (i.e., states in $\mathcal{C}^{2} \otimes \mathcal{C}^{2}$ ) and one qubit and one qutrit (i.e., states in $\mathcal{C}^{2} \otimes \mathcal{C}^{3}$ ) [8], the situation is particularly simple in the case of small $K$. For $K=1$ there exists only one state (a singlet). For $K=2$ we will present below a necessary and sufficient condition for a given mixed state to have a Slater number of 1 . One should note, however, that in the considered case of fermionic states there exists no simple analogy of the partial transposition, which is essential for the theory of entangled states. In fact, the Peres-Horodecki criterion $[7,8]$ in $2 \times 2$ and $2 \times 3$ spaces says that a state is separable iff its partial transpose is positive. It is known, however, that the PeresHorodecki criterion is equivalent to Wootters' result [18], relating separability to a quantity called concurrence, which is related to eigenvalues of a certain matrix. This latter approach can be used to characterize fermionic states in $\mathcal{A}\left(\mathcal{C}^{4}\right.$ $\otimes \mathcal{C}^{4}$ ). We have the following theorem.

Theorem 1. Let the mixed state acting in $\mathcal{A}\left(\mathcal{C}^{4} \otimes \mathcal{C}^{4}\right)$ have a spectral decomposition $\rho=\sum_{i=1}^{r}\left|\Psi_{i}\right\rangle\left\langle\Psi_{i}\right|$, where $r$ is the rank of $\rho$, and the eigenvectors $\left|\Psi_{i}\right\rangle$ belonging to nonzero eigenvalues $\lambda_{i}$ are normalized as $\left\langle\Psi_{i} \mid \Psi_{j}\right\rangle=\lambda_{i} \delta_{i j}$. Let $\left|\Psi_{i}\right\rangle=\Sigma_{a, b} w_{a b}^{i} f_{a}^{\dagger} f_{b}^{\dagger}|\Omega\rangle$ in some basis and define the complex symmetric $r \times r$ matrix $C$ by

$$
C_{i j}=\sum_{a b c d} \epsilon^{a b c d} w_{a b}^{i} w_{c d}^{j}
$$

which can be represented using a unitary matrix as $C$ $=U C_{d} U^{T}$, with $C_{d}=\operatorname{diag}\left[c_{1}, c_{2}, \cdots, c_{r}\right]$ diagonal and $\left|c_{1}\right|$ $\geqslant\left|c_{2}\right| \geqslant \cdots \geqslant\left|c_{r}\right|$. The state $\rho$ has Slater number 1 iff

$$
\left|c_{1}\right| \leqslant \sum_{i=2}^{r}\left|c_{i}\right|
$$

Proof. Let us assume that a state $\rho$ acting in $\mathcal{A}\left(\mathcal{C}^{4} \otimes \mathcal{C}^{4}\right)$ has Slater number 1, i.e.,

$$
\rho=\sum_{i=1}^{r}\left|\Psi_{i}\right\rangle\left\langle\Psi_{i}\left|=\sum_{k=1}^{r^{\prime}}\right| \phi_{k}\right\rangle\left\langle\phi_{k}\right|,
$$

where all $\phi_{k}$ have Slater rank 1, whereas $r^{\prime}$ can be an arbitrary integer $\geqslant r$. But $\left|\phi_{k}\right\rangle$ can be represented as $\left|\phi_{k}\right\rangle$ $=\sum_{i=1}^{r} U_{k i}\left|\Psi_{i}\right\rangle=\sum_{i=1}^{r} \Sigma_{a, b} U_{k i} w_{a b}^{i} f_{a}^{\dagger} f_{b}^{\dagger}|\Omega\rangle$. From lemma 3 we obtain that for each $k, \eta\left(w^{\prime}(k)\right)=0$, where $w^{\prime}(k)_{a b}$ $=\sum_{i=1}^{r} U_{k i} w_{a b}^{i}$. The matrices $U_{k i}$ must therefore fulfill, for every $k$,

$$
\sum_{a b c d} \sum_{j=1}^{r} \epsilon^{a b c d} w_{a b}^{i} w_{c d}^{j} U_{k i} U_{k j}=\sum_{i, j=1}^{r} C_{i j} U_{k i} U_{k j}=0 .
$$

On the other hand, from Eq. (13) we obtain

$$
\sum_{k=1}^{r^{\prime}} U_{k i} U_{k j}^{*}=\delta_{i j} .
$$

The Slater rank 1 is thus equivalent to the existence of the $r^{\prime} \times r$ matrix $U_{k i}$ that fulfills Eqs. (14) and (15). It is convenient to represent the rows of the matrix $U_{k i}$ as vectors $\left|R_{k}\right\rangle$ in an $r$ dimensional Hilbert space $\mathcal{H}_{\text {aux }}$. Equations (14) and (15) then reduce to $\Sigma_{k}^{r^{\prime}}\left|R_{k}\right\rangle\left\langle R_{k}\right|=1$, and $\left\langle R_{k}^{*}|C| R_{k}\right\rangle=0$ for all $k$. One can always change the basis in $\mathcal{H}_{\text {aux }}$, i.e., replace $\left|R_{k}\right\rangle \rightarrow U\left|R_{k}\right\rangle$. Such a transformation does not affect Eq. (15) and transforms $C \rightarrow U^{T} C U$. Since $C$ is symmetric, $U$ can be chosen in such a way that $U^{T} C U$ is diagonal, and Eq. (14) reads then $\sum_{i=1}^{r} c_{i} U_{k i}^{2}=0$. In this new basis the construction of $U_{k i}$ using the method of Wootters [18] can be carried over. One can always assume that $c_{1} U_{k 1}^{2}$ is real and positive by chosing the phases of $\left|R_{k}\right\rangle$. Then one observes that, provided Eq. (14) is fulfilled,

$$
0=\left|\sum_{i=1}^{r} c_{i} U_{k i}^{2}\right| \geqslant\left|c_{i}\right|\left|U_{k 1}^{2}\right|-\sum_{i=2}^{r}\left|c_{i}\right|\left|U_{k i}^{2}\right| .
$$

Summing the above inequality over $k$ and using Eq. (15), we obtain the necessary condition

$$
\left|c_{1}\right| \leqslant \sum_{i=2}^{r}\left|c_{i}\right|
$$

To show that it is also a sufficient condition, we take $r^{\prime}=2$ if $r=2, \quad r^{\prime}=4$ if $r=3,4, \quad r^{\prime}=8$ if $r=5,6, \quad$ and $U_{k i}=$ $\pm 1_{k i} \exp \left(i \theta_{i}\right) / \sqrt{r^{\prime}}$. The equations in Eq. (14) are then all equivalent to

$$
\left|c_{1}\right|=\sum_{i=2}^{r} c_{i} \exp \left(2 i \theta_{i}\right)
$$

and the angles $\theta_{i}$ can indeed be chosen to assure that Eq. (18) is fulfilled, provided the condition (17) holds. The $\pm 1_{k i}$ signs are designed in such a way that Eq. (15) is fulfilled. Thus for $r^{\prime}=2$ we take $(++),(+-)$ for $i=1,2$, for $r^{\prime}=4$ we take $(++++),(++--),(+-+-),(+--+)$ for $i$ $=1, \ldots, 4$ (or any 3 of them for $i=1, \ldots, 3$ ), and finally for $r^{\prime}=8, \quad(++++++++), \quad(++++----)$, $(++--++--), \quad(++----++), \quad(+-+-+-+-)$, $(+-+--+-+)$. In the latter case we take again as many vectors as we need, i.e., $i=1, \ldots, 5 \leqslant r \leqslant 6$.

The above theorem is an analog of the Peres-HorodeckiWootters result for two-fermion systems having a singleparticle space of dimension $2 K \leqslant 4$. The situation is much 
more complicated when we go to $K>2$; this is similar to the case of the separability problem in $\mathcal{C}^{M} \otimes \mathcal{C}^{N}$ with $M N>6$. These issues are investigated in Sec. V. In the following section, however, we shall concentrate on the case $K=2$.

\section{SLATER CORRELATION MEASURE}

The similarity of our approach to that of Wootters [18] can be pushed further and, in particular, allows us to define and calculate, for the case of $K=2$, the "Slater formation measure" (in analogy to entanglement formation measure [19]).

To this aim we first consider a pure (normalized) state $|\bar{\psi}\rangle=\Sigma_{a, b} w_{a b} f_{a}^{\dagger} f_{b}^{\dagger}|\Omega\rangle$ and define the Slater correlation measure of $|\bar{\psi}\rangle$ as in lemma 3 (cf. Ref. [13]),

$$
\eta(|\bar{\psi}\rangle)=|\langle\tilde{\psi} \mid \bar{\psi}\rangle|
$$

with $|\tilde{\bar{\psi}}\rangle$ being the dual of $|\bar{\psi}\rangle$. Obviously, the notion of dual states, as well as the function $\eta(\cdot)$ in Eq. (19), can be defined also for unnormalized states. In the following we will denote such unnormalized states just as states occurring in the previous sections, i.e., without the overbar.

The measure (19) has all desired properties [19,23], such that it vanishes iff $|\bar{\psi}\rangle$ has Slater rank 1 and it is invariant with respect to local bilateral unitary operations or, in another words, with respect to changes of the basis in the single-particle space.

Having defined the measure for the pure states, we can consider the following definition

Definition 2. Consider a density matrix $\rho$ acting in $\mathcal{A}\left(\mathcal{C}^{4} \otimes \mathcal{C}^{4}\right)$ and all its possible convex decompositions in terms of pure states, i.e., $\rho=\Sigma_{i}\left|\psi_{i}\right\rangle\left\langle\psi_{i}\left|=\Sigma_{i} p_{i}\right| \bar{\psi}_{i}\right\rangle\left\langle\bar{\psi}_{i}\right|$, where the unnormalized states $\left|\psi_{i}\right\rangle=\sqrt{p_{i}}\left|\bar{\psi}_{i}\right\rangle$; the Slater correlation measure of $\rho, \mathcal{C}_{\mathrm{SI}}(\rho)$, is defined as

$$
\mathcal{C}_{\mathrm{Sl}}(\rho)=\inf \left\{\sum_{i} p_{i} \eta\left(\left|\bar{\psi}_{i}\right\rangle\right)\right\},
$$

where the infimum is taken over all decompositions.

In other words, $\mathcal{C}_{\mathrm{Sl}}(\rho)$ is the minimal amount of Slater correlations of the pure states that are needed in order to construct $\rho$, and there is a construction of $\rho$ that uses pure states with "averaged" Slater correlation $\mathcal{C}_{\mathrm{Sl}}(\rho)$.

Note that $\Sigma_{i} p_{i} \eta\left(\left|\bar{\psi}_{i}\right\rangle\right)=\Sigma_{i} \eta\left(\left|\psi_{i}\right\rangle\right)$. As we shall see below, the measure $\mathcal{C}_{\mathrm{Sl}}(\rho)$ can be related directly to the matrix $C_{i j}$ in Eq. (11) and to its "concurrence." It is invariant not only with respect to local bilateral unitary operations, but it also cannot increase under local bilateral operations. These are trace preserving maps of the form $\rho \rightarrow M(\rho)$ $=\sum_{j} A_{j} \otimes A_{j} \rho A_{j}^{\dagger} \otimes A_{j}^{\dagger}$, where each $A_{j}$ acts in $\mathcal{C}^{4}$, and $\sum_{j} A_{j}^{\dagger} A_{j} \otimes A_{j}^{\dagger} A_{j}=1$. Such transformations correspond to mixtures of density matrices obtained after nonunitary changes of the basis in the single-particle space. It is easy to see that

$$
\mathcal{C}_{\mathrm{Sl}}(M(\rho))=\left(\sum_{j}\left|\operatorname{det} A_{i}\right| \mathcal{C}_{\mathrm{Sl}}(\rho)\right) \leqslant \mathcal{C}_{\mathrm{Sl}}(\rho)
$$

We have the following theorem

Theorem 2. For any $\rho$ acting in $\mathcal{A}\left(\mathcal{C}^{4} \otimes \mathcal{C}^{4}\right)$,

$$
\mathcal{C}_{\mathrm{Sl}}(\rho)=\left|c_{1}\right|-\sum_{i=2}^{r^{\prime}}\left|c_{i}\right|
$$

where $c_{i}$ are the diagonal elements of $C[E q .(11)]$ in the basis that diagonalizes it.

Proof. The proof is essentially the same as the one in the previous section. Let us consider an arbitrary expansion of a given density matrix, $\rho=\Sigma_{k=1}^{r^{\prime}}\left|\phi_{k}\right\rangle\left\langle\phi_{k}\right|$, where $\left|\phi_{k}\right\rangle$ $=\sum_{j=1}^{r} U_{k j}\left|\Psi_{j}\right\rangle$. Here $\left|\Psi_{j}\right\rangle$ denote the usual "subnormalized" eigenvectors of $\rho$ with $\left\langle\Psi_{j} \mid \Psi_{j}\right\rangle$ being equal to the $j$ th nonzero eigenvalue of $\rho$ [18]. It is easy to see that

$$
\mathcal{C}_{\mathrm{SI}}\left(\left|\phi_{k}\right\rangle\left\langle\phi_{k}\right|\right)=\left|\sum_{i, j=1}^{r} \mathcal{C}_{i j} U_{k i} U_{k j}\right|,
$$

and $\sum_{k=1}^{r^{\prime}} U_{k i}^{*} U_{k j}=\delta_{i j}$. By changing the basis to the one in which $C$ is diagonal, we get (after choosing the phases of $U_{k 1}$ such that $c_{1} U_{k 1}^{2}$ are real and positive)

$$
\sum_{k=1}^{r^{\prime}} \mathcal{C}_{\mathrm{SI}}\left(\left|\phi_{k}\right\rangle\left\langle\phi_{k}\right|\right)=\sum_{k=1}^{r^{\prime}}\left|\sum_{j} c_{j} U_{k j}^{2}\right| \geqslant\left|c_{1}\right|-\sum_{i=2}^{r^{\prime}}\left|c_{i}\right| .
$$

This inequality becomes an equality when we use the same construction of $U_{k j}$ as in previous section, namely, $U_{k j}=$ $\pm 1_{k j} \exp \left(i \theta_{j}\right) / \sqrt{r^{\prime}}$, with $\theta_{j}$ selected in such a way that (independently of $k$ )

$$
\left|\sum_{j} c_{j} U_{k j}^{2}\right|=\frac{1}{r^{\prime}}\left(\left|c_{1}\right|-\sum_{i=2}^{r^{\prime}}\left|c_{i}\right|\right) .
$$

The above construction provides, to our knowledge, a rare example of an analog of the entanglement formation measure that can be evaluated analytically. Obviously, since we have introduced the concept of Slater coefficients, we may define other Slater correlations measures for pure states in terms of appropriately designed convex functions of the Slater coefficients (in analogy to entanglement monotones [24]). For $K$ $=2$ and most probably only for $K=2$, all those measures are equivalent and the corresponding induced measures for mixed states can be calculated analytically.

\section{SLATER WITNESSES}

We now investigate fermion systems with single-particle Hilbert spaces of dimension $2 K>4$. In this case, a full and explicit characterization of pure and mixed state quantum correlations, such as given above for the two-fermion system with $K=2$, is apparently not possible. Therefore one has to formulate other methods to investigate the Slater number of a given state. We can, however, follow here the lines of the papers that we have written on entanglement witnesses $[10,16]$ and Schmidt number witnesses [17].

In order to determine the Slater number of a density ma- 
trix $\rho$, we note that due to the fact that the sets $\mathrm{Sl}_{k}$ are convex and compact, any density matrix of class $k$ can be decomposed into a convex combination of a density matrix of class $k-1$, and a remainder $\delta[25]$.

Proposition 1. Any state of class $k, \rho_{k}$, can be written as a convex combination of a density matrix of class $k-1$ and a so-called $k$-edge state $\delta$ :

$$
\rho_{k}=(1-p) \rho_{k-1}+p \delta, \quad 1 \geqslant p>0,
$$

where the edge state $\delta$ has Slater number $\geqslant k$.

The decomposition (24) is obtained by subtracting projectors onto pure states of Slater rank smaller than $k, P$ $=\left|\psi^{<k}\right\rangle\left\langle\psi^{<k}\right|$, such that $\rho_{k}-\lambda P \geqslant 0$. Here $\left|\psi^{<k}\right\rangle$ stands for pure states of Slater rank $r<k$. Denoting by $K(\rho), R(\rho)$, and $r(\rho)$ the kernel, range, and rank of $\rho$, respectively, we observe that $\rho^{\prime} \propto \rho-\lambda\left|\psi^{<k}\right\rangle\left\langle\psi^{<k}\right|$ is non negative iff $\left|\psi^{<k}\right\rangle$ $\in R(\rho)$ and $\lambda \leqslant\left\langle\psi^{<k}\left|\rho^{-1}\right| \psi^{<k}\right\rangle^{-1}$ (see [25]). The idea behind this decomposition is that the edge state $\delta$ which has generically lower rank contains all the information concerning the Slater number $k$ of the density matrix $\rho_{k}$.

As in the case of Schmidt number, there is an optimal decomposition of the form (24) with $p$ minimal. Alternatively, restricting ourselves to decompositions $\rho_{k}$ $=\sum_{i} p_{i}\left|\psi_{i}^{r_{i}}\right\rangle\left\langle\psi_{i}^{r_{i}}\right|$ with all $r_{i} \leqslant k$, we can always find a decomposition of the form (24) with $\delta \in S l_{k}$. We define below more precisely what an edge state is.

Definition 3. A $k$-edge state $\delta$ is a state such that $\delta$ $-\epsilon\left|\psi^{<k}\right\rangle\left\langle\psi^{<k}\right|$ is not positive, for any $\epsilon>0$ and $\left|\psi^{<k}\right\rangle$.

Criterion 1. A mixed state $\delta$ is a $k$-edge state iff there exists no $\left|\psi^{<k}\right\rangle$ such that $\left|\psi^{<k}\right\rangle \in R(\delta)$.

Now we are in the position of defining a $k$-class Slater witness $(k-\mathrm{SW}, k \geqslant 2)$ :

Definition 4. A Hermitian operator $W$ is a Slater witness (SW) of class $k$ iff $\operatorname{Tr}(W \sigma) \geqslant 0$ for all $\sigma \in \mathrm{Sl}_{k-1}$ and there exists at least one $\rho \in \mathrm{Sl}_{k}$ such that $\operatorname{Tr}(W \rho)<0$.

It is straightforward to see that every SW that detects $\rho$ given by Eq. (24) also detects the edge state $\delta$, since if $\operatorname{Tr}(W \rho)<0$, then necessarily $\operatorname{Tr}(W \delta)<0$, too. Thus knowledge of all SW's of $k$-edge states fully characterizes all $\rho$ $\in \mathrm{Sl}_{k}$. Below, we show how to construct for any edge state a SW which detects it. Most of the technical proofs used to construct and optimize Slater witnesses are very similar to those presented in Ref. [10] for entanglement witnesses.

All the operators we consider below act in $\mathcal{A}\left(\mathcal{C}^{2 K} \otimes \mathcal{C}^{2 K}\right)$. Let $\delta$ be a $k$-edge state, $C$ an arbitrary positive operator such that $\operatorname{Tr}(\delta C)>0$, and $P$ a positive operator whose range fulfills $R(P)=K(\delta)$. We define $\epsilon$ $\equiv \inf _{\left|\psi^{<k}\right\rangle}\left\langle\psi^{<k}|P| \psi^{<k}\right\rangle$ and $c \equiv \sup \langle\psi|C| \psi\rangle$. Note that $c$ $>0$ by construction and $\epsilon>0$, because $R(P)=K(\delta)$, and therefore, since $R(\delta)$ does not contain any $\left|\psi^{<k}\right\rangle$ by the definition of edge state, $K(P)$ cannot contain any $\left|\psi^{<k}\right\rangle$ either. This implies the following.

Lemma 4. Given a $k$-edge state $\delta$, then

$$
W=P-\frac{\epsilon}{c} C
$$

is a $k$-SW which detects $\delta$.
The simplest choice of $P$ and $C$ consists in taking projections onto $K(\delta)$ and the identity operator on the asymmetric space $\mathbb{1}_{a}$, respectively. As we will see below, this choice provides us with a canonical form of a $k-\mathrm{SW}$.

Proposition 2. Any Slater witness can be written it the canonical form

$$
W=\widetilde{W}-\epsilon \mathbb{1}_{a}
$$

such that $R(\widetilde{W})=K(\delta)$, where $\delta$ is a $k$-edge state and $0<\epsilon$ $\leqslant \inf _{|\psi\rangle \in S_{k-1}}\langle\psi|\widetilde{W}| \psi\rangle$.

Proof. Assume $W$ is an arbitrary $k$-SW such that $\operatorname{Tr}(W \sigma) \geqslant 0$ for all $\sigma \in \mathrm{Sl}_{k-1}$ and there $\exists$ at least one $\rho$ such that $\operatorname{Tr}(W \rho)<0$. Here $W$ has at least one negative eigenvalue. Construct $W+\epsilon \mathbb{1}_{a}=\widetilde{W}$, such that $\widetilde{W}$ is a positive operator on $\mathcal{A}\left(\mathcal{C}^{2 K} \otimes \mathcal{C}^{2 K}\right)$, but does not have a full rank, $K(\widetilde{W}) \neq 0$ (by continuity this construction is always possible). But $\left\langle\psi^{<k}|\widetilde{W}| \psi^{<k}\right\rangle \geqslant \epsilon>0$ since $W$ is a $k$-SW, ergo no $\left|\psi^{<k}\right\rangle \in K(\widetilde{W})$.

Definition 5. A $k$-class Slater witness $W$ is tangent to $\mathrm{Sl}_{k-1}$ at $\rho$ if $\exists$ a state $\rho \in \mathrm{Sl}_{k-1}$ such that $\operatorname{Tr}(W \rho)=0$.

Observation 1. The state $\rho$ is of Slater class $k-1$ iff for all $k$-SW's tangent to $\mathrm{Sl}_{k-1}, \operatorname{Tr}(W \rho) \geqslant 0$.

Proof (see [10]). (only if) Suppose that $\rho$ is of class $k$. From the Hahn-Banach theorem it follows that there exists a $k$-SW, $W$, that detects it. We can subtract $\epsilon \mathbb{1}_{a}$ from $W$, making $W-\epsilon \mathbb{1}_{a}$ tangent to $\mathrm{Sl}_{k-1}$ at some $\sigma$, but then $\operatorname{Tr}[\rho(W$ $-\epsilon \mathbb{1})]<0$.

\section{A. Optimal Slater witnesses}

We will now discuss the optimization of a Slater witness. As proposed in [10] and [17], an entanglement witness (Schmidt witness) $W$ is optimal if there exists no other witness that detects more states than it. The same definition can be applied to Slater witnesses. We say that a $k$-Slater witness $W_{2}$ is finer than a $k$-Slater witness $W_{1}$, if $W_{2}$ detects more states than $W_{1}$. Analogously, we define a $k$-Slater witness $W$ to be optimal when there exists no finer witness than itself. Let us define the set of $\left|\psi^{<k}\right\rangle$ pure states of Slater rank $k$ -1 for which the expectation value of the $k$-Slater witness $W$ vanishes:

$$
T_{W}=\left\{\left|\psi^{<k}\right\rangle \quad \text { such that }\left\langle\psi^{<k}|W| \omega^{<k}\right\rangle=0\right\},
$$

i.e., the set of pure tangent states of Slater rank $<k$. Here $W$ is an optimal $k-\mathrm{SW}$ iff $W-\epsilon P$ is not a $k-\mathrm{SW}$, for any positive operator $P$. If the set $T_{W}$ spans the whole Hilbert space $\mathcal{A}\left(\mathcal{C}^{2 K} \otimes \mathcal{C}^{2 K}\right)$, then $W$ is an optimal $k$-SW. If $T_{W}$ does not span $\mathcal{A}\left(\mathcal{C}^{2 K} \otimes \mathcal{C}^{2 K}\right)$, then we can optimize the witness by subtracting from it a positive operator $P$, such that $P T_{W}=0$. For example, for Slater witnesses of class 2 this is possible pro-

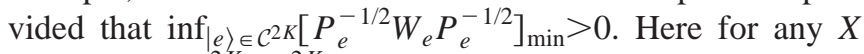
acting on $\mathcal{A}\left(\mathcal{C}^{2 K} \otimes \mathcal{C}^{2 K}\right)$, we define

$$
\begin{aligned}
X_{e}= & {[\langle e, \cdot|X| e, \cdot\rangle-\langle e, \cdot|X| \cdot, e\rangle-\langle\cdot, e|X| e, \cdot\rangle} \\
& +\langle\cdot, e|X| \cdot, e\rangle],
\end{aligned}
$$


as an operator acting in $\mathcal{C}^{2 K}$, and $[X]_{\min }$ denotes its minimal eigenvalue (see [10]). An example of an optimal witness of Slater number $k$ in $\mathcal{A}\left(\mathcal{C}^{2 K} \otimes \mathcal{C}^{2 K}\right)$ is given by

$$
W=\mathbb{1}_{a}-\frac{K}{k-1} \mathcal{P},
$$

where $\mathcal{P}$ is a projector onto a "maximally correlated state," $|\Psi\rangle=(1 / \sqrt{K}) \sum_{i=1}^{K} f_{a_{1}(i)}^{\dagger} f_{a_{2}(i)}^{\dagger}|\Omega\rangle$ [cf. Eq. (5)]. The reader can easily check that the above witness operator has mean value zero in the states $f_{a_{1}(i)}^{\dagger} f_{a_{2}(i)}^{\dagger}|\Omega\rangle$ for $i=1,2$, but also for all states of the form $g_{1}^{\dagger} g_{2}^{\dagger}|\Omega\rangle$, where

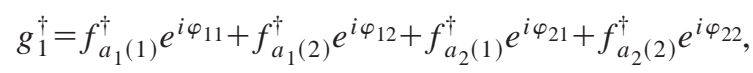

$$
\begin{aligned}
& g_{2}^{\dagger}=-f_{a_{2}(1)}^{\dagger} e^{-i \varphi_{12}}+f_{a_{1}(2)}^{\dagger} e^{-i \varphi_{11}}-f_{a_{2}(1)}^{\dagger} e^{-i \varphi_{22}} \\
& +f_{a_{2}(2)}^{\dagger} e^{-i \varphi_{21}},
\end{aligned}
$$

for arbitrary $\varphi_{i j}, i, j=1,2$. The set $T_{W}$ spans in this case the whole Hilbert space $\mathcal{A}\left(\mathcal{C}^{2 K} \otimes \mathcal{C}^{2 K}\right)$ : ergo $W$ is optimal.

\section{B. Slater witnesses and positive maps}

It is interesting to consider linear maps associated with Slater witnesses via the Jamiołkowski isomorphism [21]. Such maps employ $W$ acting in $\mathcal{H}_{A} \otimes \mathcal{H}_{B}=\mathcal{C}^{2 K} \otimes \mathcal{C}^{2 K}$ and transform a state $\rho$ acting in $\mathcal{H}_{A} \otimes \mathcal{H}_{C}=\mathcal{C}^{2 K} \otimes \mathcal{C}^{2 K}$ into another state acting in $\mathcal{H}_{B} \otimes \mathcal{H}_{C}=\mathcal{C}^{2 K} \otimes \mathcal{C}^{2 K}, \quad M(\rho)$ $=\operatorname{Tr}_{A}\left(W \rho_{A}^{T}\right)$. Obviously, such maps are positive on separable states: When $\rho$ is separable, then for any $|\Psi\rangle \in \mathcal{H}_{B}$ $\otimes \mathcal{H}_{\mathcal{C}}$, the mean value of $\langle\Psi|M(\rho)| \Psi\rangle$, becomes a convex sum of mean values of $W$ in some product states $|e, f\rangle$ $\in \mathcal{H}_{A} \otimes \mathcal{H}_{B}$. Since $W$ acts in fact in the antisymmetric space, we can antisymmetrize these states, i.e., $|e, f\rangle \rightarrow(|e, f\rangle$ $-|f, e\rangle)$. Such antisymmetric states have, however, Slater rank 1 , and all SW's-of class $k \geqslant 2$ have thus positive mean value in those states. This class of positive maps is quite different from the ones considered in Refs. [10], [16]; they provide thus an interesting class of necessary separability conditions. The map associated with the witness (29) is, however, decomposable; i.e., it is a sum of a completely positive map and another completely positive map composed with transposition. This follows from the fact that the witness operator has a positive partial transpose; i.e., it can be presented as a partial transpose of a positive operator.

\section{CONCLUSIONS AND OUTLOOK}

Summarizing, we have presented a general characterization of quantum correlated states in two-fermion systems with a $2 K$-dimensional single-particle space. This goal has been achieved by introducing the concepts of Slater deomposition and rank for pure states, and Slater number for mixed states. In particular, for the important case $K=2$ the quantum correlations in mixed states can be characterized completely in analogy to Wootters' result for separated qubits [18] and using the findings of Ref. [13] for pure states. Simi- larly to the case of separated systems, the situation for $K$ $>2$ is more complicated. Therefore, we have also introduced witnesses of Slater number $k$ and presented the methods of optimizing them.

Possible directions for future work include generalizations of the present results to more than two fermions and the development of an analogous theory for indistinguishable bosons. For this purpose a lot of the concepts developed so far are expected to be useful there as well. However, there are certainly also fundamental differences between quantum correlations in bosonic and fermionic systems. As an example, consider the notion of unextendible product bases introduced recently for separated systems [26]. These are sets of product states spanning a subspace of the Hilbert space whose orthogonal complement does not contain any product states. All such unextendible product bases constructed so far involve product states of the form $|\psi\rangle \otimes|\chi\rangle$ with $|\psi\rangle$ and $|\chi\rangle$ being nonorthogonal. In the analogous fermionic state nonorthogonal contributions are obviously cancelled out by antisymmetrization, unlike the bosonic case. In fact, all explicit constructions of unextendible product bases known so far [26] can be taken over directly to bosonic systems to give "unextendible Slater permanent bases." These are sets of symmetrized product states spanning a subspace of the symmetrized Hilbert space, whose orthogonal complement does not contain any such states.

\section{ACKNOWLEDGMENTS}

We thank Anna Sanpera and W. K. Wootters for useful discussions, and Allan H. MacDonald for helpful comments and a critical reading of the manuscript. J.S. was supported by the Deutsche Forschungsgemeinschaft under Grant No. SCHL 539/1-1. M.K. was supported by Polish KBN Grant No. 2 P03B 072 19. M.L. acknowledges support by the Deutsche Forschungsgemeinschaft via SFB 407, Schwerpunkt "Quanteninformationsverarbeitung," and the Projekt 436 POL 133/86/0. D.L. acknowledges partial support from the Swiss National Science Foundation.

\section{APPENDIX}

We now list further properties of the correlation measure $\eta$ for pure states $|\Psi\rangle=\Sigma_{a, b=1}^{4} w_{a b} f_{a}^{\dagger} f_{b}^{\dagger}|\Omega\rangle$ of two fermions in a four-dimensional single-particle space [13] and add some further remarks.

The matrix $w$ transforms under a unitary transformation of the one-particle space,

$$
f_{a}^{\dagger} \mapsto \mathcal{U} f_{a}^{\dagger} \mathcal{U}^{\dagger}=\sum_{b} U_{b a} f_{b}^{\dagger},
$$

as

$$
w \mapsto U w U^{T},
$$

where $U^{T}$ is the transpose (not the adjoint) of $U$. Under such a transformation, $|\Psi\rangle \mapsto|\Phi\rangle=\mathcal{U}|\Psi\rangle$, scalar products of the form $\left\langle\widetilde{\Psi}_{1} \mid \Psi_{2}\right\rangle$ remain unchanged up to a phase, 


$$
\left\langle\widetilde{\Phi}_{1} \mid \Phi_{2}\right\rangle=\operatorname{det} U\left\langle\widetilde{\Phi}_{1} \mid \Phi_{2}\right\rangle .
$$

Therefore, in particular, $\eta(|\Psi\rangle)$ is invariant under arbitrary single-particle transformations.

The dualization of a state $|\Psi\rangle$ can be identified as a particle-hole-transformation,

$$
\mathcal{U}_{p-h} f_{a}^{\dagger} \mathcal{U}_{p-h}^{\dagger}=f_{a}, \quad \mathcal{U}_{p-h}|\Omega\rangle=f_{1}^{\dagger} f_{2}^{\dagger} f_{3}^{\dagger} f_{4}^{\dagger}|\Omega\rangle,
$$

along with a complex conjugation. In fact, the operator of dualization $\mathcal{D},|\Psi\rangle \mapsto|\widetilde{\Psi}\rangle=\mathcal{D}|\Psi\rangle$, can be written as

$$
\mathcal{D}=-\mathcal{U}_{p-h} \mathcal{K},
$$

where $\mathcal{K}$ is the usual operator of complex conjugation which acts on a general state vector as

$$
\mathcal{K}(a|\alpha\rangle+b|\beta\rangle)=a^{*} \mathcal{K}|\alpha\rangle+b^{*} \mathcal{K}|\beta\rangle .
$$

Its action on the single-particle basis states and the fermionic vacuum is given by

$$
\mathcal{K} f_{a}^{\dagger} \mathcal{K}=f_{a}^{\dagger}, \quad \mathcal{K} f_{a} \mathcal{K}=f_{a}, \quad \mathcal{K}|\Omega\rangle=|\Omega\rangle .
$$

The relations (A7) are to be seen as a part of the definition of $\mathcal{K}$ and refer explicitly to a certain single-particle basis defined by the operators $f_{a}, f_{a}^{\dagger}$. However, switching to a different complex conjugation operator $\mathcal{K}^{\prime}$, fulfilling the relations (A7) in a different basis, has only trivial effects without any physical significance. In particular, as one can see from the properties given above, the correlation measure $\eta(|\Psi\rangle)$ $=|\langle\widetilde{\Psi} \mid \Psi\rangle|,|\widetilde{\Psi}\rangle=\mathcal{D}|\Psi\rangle$, remains invariant under such an operation.
Equation (A3) implies that $\mathcal{D}$ is unchanged by unitary single-particle operations,

$$
\mathcal{U} \mathcal{D} \mathcal{U}^{\dagger}=\mathcal{D} \Leftrightarrow[\mathcal{U}, \mathcal{D}]=0
$$

which can also be expressed as

$$
\mathcal{U}_{p-h} \mathcal{U}^{T}=\mathcal{U}_{p-h}
$$

for any unitary single-particle transformation $\mathcal{U}$.

The dualization operator $\mathcal{D}$ is the antiunitary implementation of the particle-hole transformation. We note that the complex conjugation involved there is necessary for $\mathcal{D}$ being compatible with single-particle transformations $\mathcal{U}$,

$$
\mathcal{D} \mathcal{U} f_{a}^{\dagger} \mathcal{U}^{\dagger} \mathcal{D}^{-1}=\sum_{b} U_{b a}^{*} f_{b}=\mathcal{U} \mathcal{D} f_{a}^{\dagger} \mathcal{D}^{-1} \mathcal{U}^{\dagger}
$$

If the complex conjugation would be left out, $\mathcal{U}$ and $\mathcal{D}$ would not commute.

The relation of the correlation measure $\eta$ to an antiunitary operator is similar to Wootters' construction for a separate system of two qubits [18]. The correlation measure there ("concurrence") relies on the time inversion operation. The operator of time inversion in the two-qubit system is invariant under local unitary transformations in each qubit space. This property is similar to the invariance of the dualization operator under unitary transformations in the single-particle space.
[1] For a review see M. Horodecki, P. Horodecki, and R. Horodecki, in Quantum Information-Basic Concepts and Experiments, edited by G. Alber and M. Weiner (Springer, Berlin, in press).

[2] For a primer see M. Lewenstein, D. Bruß, J. I. Cirac, M. Kus, J. Samsonowicz, A. Sanpera, and R. Tarrach, J. Mod. Opt. 77, 2481 (2000).

[3] The Physics of Quantum Information, edited by D. Bouwmeester, A. Ekert, and A. Zeilinger (Springer, Heidelberg, 2000).

[4] A. K. Ekert, Phys. Rev. Lett. 67, 661 (1991); C. H. Bennett and S. J. Wiesner, ibid. 69, 2881 (1992); C. H. Bennett, G. Brassard, S. Popescu, B. Schumacher, J. A. Smolin, and W. K. Wootters, ibid. 76, 722 (1996).

[5] R. F. Werner, Phys. Rev. A 40, 4277 (1989).

[6] A. Peres, Quantum Theory: Concepts and Methods (Kluwer Academic, Dordrecht, 1995).

[7] A. Peres, Phys. Rev. Lett. 77, 1413 (1996).

[8] M. Horodecki, P. Horodecki, and R. Horodecki, Phys. Lett. A 223, 8 (1996).

[9] B. M. Terhal, Linear Algebr. Appl. 323, 61 (2000); Phys. Lett. A 271, 319 (2000).

[10] M. Lewenstein, B. Kraus, J. I. Cirac, and P. Horodecki, Phys. Rev. A 62, 052310 (2000).
[11] D. Loss and D. P. DiVincenzo, Phys. Rev. A 57, 120 (1998).

[12] G. Burkard, D. Loss, and D. P. DiVincenzo, Phys. Rev. B 59, 2070 (1999).

[13] J. Schliemann, D. Loss, and A. H. MacDonald, Phys. Rev. B 63, 085311 (2001).

[14] D. Jaksch, H.-J. Briegel, J. I. Cirac, C. W. Gardiner, and P. Zoller, Phys. Rev. Lett. 82, 1975 (1999); H.-J. Briegel, T. Calarco, D. Jaksch, J. I. Cirac, and P. Zoller, J. Mod. Opt. 47, 415 (2000).

[15] G. Birkl, F. B. J. Buchkremer, R. Dumke, and W. Ertmer, Opt. Commun. 191, 67 (2001).

[16] M. Lewenstein, B. Kraus, P. Horodecki, and J. I. Cirac, Phys. Rev. A 63, 044304 (2001).

[17] A. Sanpera, D. Bruß, and M. Lewenstein, Phys. Rev. A 63, 050301 (2001).

[18] W. K. Wootters, Phys. Rev. Lett. 80, 2245 (1998).

[19] C. H. Bennett, D. P. DiVincenzo, J. A. Smolin, and W. K. Wootters, Phys. Rev. A 54, 3824 (1996).

[20] B. M. Terhal and P. Horodecki, Phys. Rev. A 61, 040301 (2000).

[21] A. Jamiołkowski, Rep. Math. Phys. 3, 275 (1972).

[22] M. L. Mehta, Elements of Matrix Theory (Hindustan, Delhi, 1977). Alternatively, see R. A. Horn and C. R. Johnson, Matrix 
Analysis (Cambridge University Press, Cambridge, England, 1986), theorems 2.3.4 and 2.3.6.

[23] V. Vedral and M. B. Plenio, Phys. Rev. A 57, 3 (1998).

[24] G. Vidal, J. Mod. Opt. 47, 355 (2000).

[25] M. Lewenstein and A. Sanpera, Phys. Rev. Lett. 80, 2261 (1998); A. Sanpera, R. Tarrach, and G. Vidal, Phys. Rev. A 58, 826 (1998).
[26] C. H. Bennett, D. P. DiVincenzo, T. Mor, P. W. Shor, J. A. Smolin, and B. M. Terhal, Phys. Rev. Lett. 82, 5385 (1999); D. P. DiVincenzo, T. Mor, P. W. Shor, J. A. Smolin, and B. M. Terhal, Commun. Math. Phys. (to be published), e-print quant-ph/9908070; D. P. DiVincenzo and B. M. Terhal, e-print quant-ph/0008055. 